\title{
Interplay of Kondo and Fano Resonance in Electronic Transport in Nanostructures
}

\author{
B.R. BuŁka ${ }^{a}$, P. Stefański ${ }^{a}$ And A. Tagliacozzo ${ }^{b}$ \\ ${ }^{a}$ Institute of Molecular Physics, Polish Academy of Sciences \\ Smoluchowskiego 17, 60-179 Poznań, Poland \\ ${ }^{b}$ Coherentia-INFM, Unita di Napoli and Dipartimento di Scienze Fisiche \\ Universita di Napoli Federico II \\ Monte S. Angelo-via Cintia, 80126 Napoli, Italy
}

\begin{abstract}
Quantum interference and electronic correlations are processes relevant for electronic transport in nanostructures. We present theoretical studies of transport for various models, which take into account these processes and describe the Fano resonance as well as many-body effects like the Kondo resonance. The results are compared with transport measurements performed on a small quantum dot strongly coupled to electrodes, a large semi-open quantum dot, a side-attached quantum dot to a quantum wire systems and a quantum dot embedded in the metallic ring. We show how conductance characteristics of the Kondo and the Fano resonance are modified in different situations.
\end{abstract}

PACS numbers: 73.63.-b, 72.15.Qm, 75.20.Hr

\section{Introduction}

Electronic transport in nanostructures attracts recently a great interest, as it is a fundamental issue to application in electronics. In devices of a nanometer scale transport is governed by different mechanisms than those known in macroscopic devices. Electrons in such small systems exhibit their corpuscular-wave nature. Electronic waves can propagate coherently and interference processes are relevant for operation of electronic nanodevices. Coherence of electronic waves is achieved if the size of the system becomes smaller than the coherence length, which for silicon devices is estimated as $10 \mathrm{~nm}$ at the room temperature. Due to recent technological achievements many experiments were performed, in which quantum interference phenomena were observed. In this paper we want to review 
some of them. First, the Fano resonance [1] will be presented as a special kind of interference process. The Fano effect is known in various branches of condensed matter physics, and recently it was observed in transport through the quantum dots. The present nanotechnology allows production of the quantum dot (QD) systems as well-controlled objects, which offer the possibility of continuous tuning of the relevant parameters: the position of electronic spectrum, the number of electrons, the strength of the electrode-QD coupling (e.g. see [2]). It gives opportunity for a quantitative comparison of theoretical studies of the Fano resonance for various QD systems. We will present results of calculations of electronic transport in the bridge model, in the model for a side-attached quantum dot as well as in the model of a large semi-open quantum dot, which explain and describe quantitatively observed interference effects in various QD systems.

The Aharonov-Bohm effect [3] in a nanoscale metallic ring will be also considered in the paper. The first experiment was performed by Webb et al. [4] 20 years ago. It was a first milestone on the roadmap to nanoelectronics, as it showed that the wave nature of electronic transport can be relevant for operation of electronic devices in very large scale of integration. Recent studies concern electronic transport through quantum dots placed in arms of the metallic ring [5]. These experiments give opportunity to analyze interplay of quantum interference and electronic correlations, in particular to study modification of conductance characteristics due to changes of the magnetic flux in the presence of the Kondo resonance [6]. Electrons traversing the QD interact with uncompensated localized spin, which leads to resonant spin-flip processes and changes in the conductance. The Kondo resonance was extensively studied in the bulk systems, but its features are different in nanostructures, where the transport is coherent [7-10]. In this paper we focus on interplay of the Kondo resonance and the quantum interference processes (as the Fano resonance and the Aharonov-Bohm effect), and how these effects are seen in electronic transport in various nanodevices.

These problems are fundamental for applications in electronic devices operating in coherent transport regime. Electron-electron correlations have recently became a topic of broad interest, as it is essential for applications in quantum computing. The theoretical efficiency of quantum computers follows from the fact that they exploit the coherent superposition of wave functions. Spintronics is another field of interest, in which one plans to use a spin degree of freedom of electrons (instead of charge) in electronic devices. Studies of quantum interference, decoherence processes, many-body effects, charge and spin correlations are basic for these applications. The paper is devoted to some aspect of these problems.

The paper is organized as follows. First, in Sect. 2 we present main features of the Kondo resonance in a bulk system and in nanostructures, showing differences in transport and their physical nature. Next, in Sect. 3 the Fano resonance is described, its origin and features seen in transport in nanostructures. In this part interplay of both the resonances and their role in the conductance is presented for 
various models. Next, the problem of the Aharonov-Bohm effect and the Kondo resonance in the metallic ring with a quantum dot is also considered. The paper concludes with the final remarks and summary in Sect. 4.

\section{Kondo resonance}

\subsection{Main features of the Kondo resonance in a bulk system}

The main feature of the Kondo resonance is the minimum in the electrical resistivity. A first report on measurements of this effect has appeared in 30's of the last century and it was performed on Au doped by magnetic atoms in temperatures below $4 \mathrm{~K}$. The effect was mysterious up to 1964 when Kondo [6] proposed a resonant spin-flip scattering process as that one responsible for this effect. His considerations were based on the model for conducting electrons scattered by localized spins on magnetic impurities, which is given by the following Hamiltonian:

$$
H=\sum_{k, \sigma} \epsilon_{k} c_{k \sigma}^{\dagger} c_{k \sigma}+\sum_{k, k^{\prime}, \sigma, \sigma^{\prime}} J \boldsymbol{S} \cdot c_{k \sigma}^{\dagger} \sigma_{\sigma \sigma^{\prime}} c_{k^{\prime} \sigma^{\prime}}
$$

The last term describes spin-flip processes of conducting electrons on the localized spin $\boldsymbol{S}$ at the impurity. From the Boltzmann theory of the electrical transport the resistivity is expressed as

$$
\rho=\frac{m}{n e^{2}} \frac{1}{\tau}
$$

where the relaxation time is given by

$$
\frac{1}{\tau}=2 \pi c_{\mathrm{imp}} \int \frac{\mathrm{d}^{3} k}{8 \pi^{3}} \delta\left(\epsilon_{k}-\epsilon_{k^{\prime}}\right)\left|T_{k k^{\prime}}\right|^{2}\left(1-\cos \theta^{\prime}\right)
$$

where $c_{\mathrm{imp}}$ is the impurity concentration and $T$ denotes the so called $T$-matrix, whose elements depend on the angle $\theta^{\prime}$ between the vectors $k$ and $k^{\prime}$. Kondo calculated a second order spin-flip contribution to the matrix elements and determined the relaxation time

$$
\frac{1}{\tau}=\frac{3 n c_{\mathrm{imp}} J^{2} S(S+1)}{2 e^{2} \hbar E_{\mathrm{F}}}\left\{1-2 J\left[g\left(E_{\mathrm{F}}\right)+g\left(E_{\mathrm{F}}\right)^{*}\right]\right\} .
$$

Here $E_{\mathrm{F}}$ denotes the Fermi energy and $g$ is the integral

$$
g(E)=\frac{1}{N} \sum_{k} \frac{f\left(\epsilon_{k}\right)}{\epsilon_{k}-E-\mathrm{i} 0^{+}},
$$

where $f$ is the Fermi distribution function. The integral $g$ is singular as the temperature $T \rightarrow 0$ and it is responsible for the logarithmic increase in the resistivity

$$
\rho_{\text {spin }}=\frac{3 \pi m J^{2} S(S+1)}{2 e^{2} \hbar E_{\mathrm{F}}}\left[1-4 J \rho\left(E_{\mathrm{F}}\right) \ln \left(\frac{k_{\mathrm{B}} T}{W}\right)\right] .
$$

$\rho\left(E_{\mathrm{F}}\right)$ and $W$ denotes the electron density of state at the Fermi level and the width of the electronic band, respectively. We remind that in most cases the cou- 
pling is antiferromagnetic $J<0$. These calculations show that the resistance is proportional to the effective cross-section of scattered electrons (3) and it reaches its maximal value for $T=0$. It is well known that the Kondo model (1) can be obtained from the single impurity Anderson model

$$
H=\sum_{k, \sigma} \epsilon_{k} c_{k \sigma}^{\dagger} c_{k \sigma}+\sum_{\sigma} \epsilon_{0} c_{0 \sigma}^{\dagger} c_{0 \sigma}+U n_{0 \uparrow} n_{0 \downarrow}+\sum_{k, \sigma} V_{k}\left(c_{k \sigma}^{\dagger} c_{0 \sigma}+c_{0 \sigma}^{\dagger} c_{k \sigma}\right),
$$

in which the second and the third term describe electrons on the impurity including the Coulomb interactions. In order to do transformation one has to neglect charge fluctuations and to consider virtual excitations from the single occupied state within lowest order perturbation theory. The effective exchange coupling is then given by

$$
J_{k, k^{\prime}}=V_{k}^{*} V_{k^{\prime}}\left(\frac{1}{U+\epsilon_{0}-\epsilon_{k^{\prime}}}+\frac{1}{\epsilon_{k}-\epsilon_{0}}\right) .
$$

Abrikosov and Suhl showed that the resonant spin-flip processes lead to formation of a high peak in the quasi-particle density of states at the Fermi energy.

\subsection{Kondo resonance in nanostructures}

The situation is different in nanostructures, where resonant Kondo scattering of electrons leads to an increase in the conductance to the maximal value $2 e^{2} / h$ for $T \rightarrow 0$. An essential difference is that in the bulk system the transport is governed by the effective cross-section and the resistivity is proportional to inverse of the relaxation time $1 / \tau$, which is the quantity averaged over many impurities. In contrast the quantum dot can be treated as a single scatterer and the current can be determined as

$$
I=\frac{2 e}{h} \int \mathrm{d} \omega\left[f_{\mathrm{L}}(E)-f_{\mathrm{R}}(E)\right]|t(E)|^{2}
$$

where $f_{\alpha}(E)$ is the Fermi distribution function for electrons in the electrodes and $t(E)$ is the transmission for an electronic wave passing through the QD. In the linear response limit (for the source-drain voltage $V_{\mathrm{sd}} \rightarrow 0$ ) the conductance can be written as

$$
\mathcal{G}=\frac{2 e^{2}}{h} \int \mathrm{d} E\left(-\frac{\mathrm{d} f}{\mathrm{~d} E}\right)|t(E)|^{2} .
$$

The Kondo problem in nanostructures was considered by Glazman and Raikh [7], $\mathrm{Ng}$ and Lee [8], and Kawabata [9] for the single impurity Anderson model (7). In low temperatures $\mathcal{G}$ is simply expressed as

$$
\mathcal{G}=\frac{2 e^{2}}{h} \frac{4 \Gamma_{\mathrm{L}} \Gamma_{\mathrm{R}}}{\left(\Gamma_{\mathrm{L}}+\Gamma_{\mathrm{R}}\right)^{2}} \sin ^{2} \eta
$$

where $\Gamma_{\alpha}$ is the transfer rate of an electron from the left $(\alpha=\mathrm{L})$ and the right $(\alpha=\mathrm{R})$ electrode to the quantum dot. $\eta$ is a shift of the phase of the electronic 
wave scattered on the QD. Using the Friedel sum rule $\eta=\pi n / 2$, which couples the phase shift $\eta$ and the number of electrons $n$, one gets in the Kondo limit $(n \rightarrow 1)$

$$
\mathcal{G}=\frac{2 e^{2}}{h} \frac{4 \Gamma_{\mathrm{L}} \Gamma_{\mathrm{R}}}{\left(\Gamma_{\mathrm{L}}+\Gamma_{\mathrm{R}}\right)^{2}}
$$

It means that for a symmetrical coupling $\left(\Gamma_{\mathrm{L}}=\Gamma_{\mathrm{R}}\right)$ the system becomes transparent for any position of the level $\epsilon_{0} \ll E_{\mathrm{F}}$. Figure 1 presents the electronic density of states and the conductance in the quantum dot.
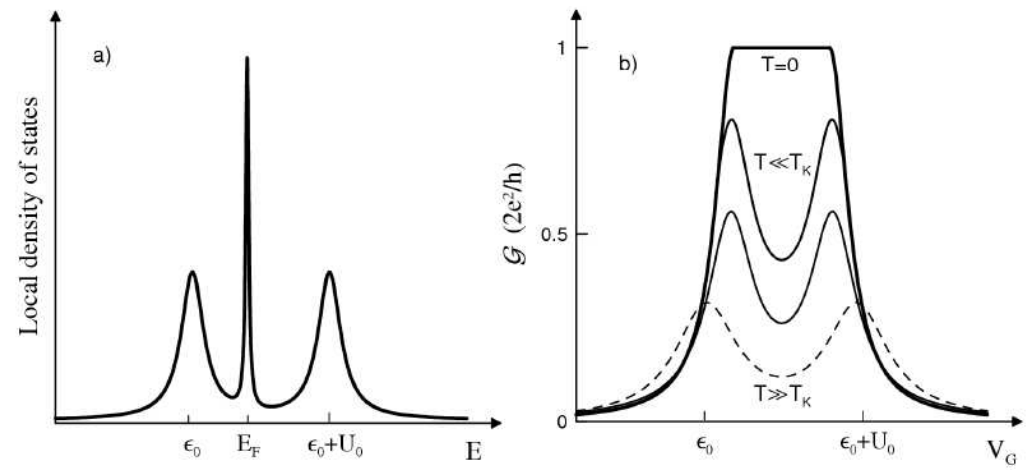

Fig. 1. (a) Schematic presentation of the local density of states at the quantum dot. The peak at $\epsilon_{0}$ and $\epsilon_{0}+U$ represent the charge fluctuations, whereas the peak at the Fermi level $E_{\mathrm{F}}$ (called the Abrikosov-Suhl peak) is due to spin-fluctuations and appears below the Kondo temperature $T_{\mathrm{K}}$. (b) Schematic presentation of the conductance $\mathcal{G}$ as a function of the gate voltage $V_{\mathrm{g}}$ for different temperatures. At $T=0$ one expects that $\mathcal{G}$ reaches its maximal value $2 e^{2} / h$ for odd numbers of electrons in the quantum dot. The dashed curve represents the conductance for high temperatures, when the Kondo resonance disappears.

In the last decade many transport measurements confirmed these predictions [7-9] on various systems: in quantum dots [11], in carbon nanotubes [12], and in single molecules [13]. The QD system is the best for studies of the Kondo resonance as one can tune experimentally parameters of the model (7): the electrodedot coupling $\Gamma_{\alpha}$ and the position of the energy level $\epsilon_{0}$. The conductance is usually plotted as a two-dimensional grey-scale map vs. the gate voltage $V_{\mathrm{g}}$ and the source-drain voltage $V_{\mathrm{sd}}$. The characteristic feature of the Kondo resonance is a maximum seen for small $V_{\mathrm{sd}}$, which is observed in small temperatures for any value $V_{\mathrm{g}}$ when the dot has odd number of electrons and uncompensated spin.

\section{Fano resonance in nanostructures}

\subsection{Quantum mechanical origin of the Fano resonance}

The Fano resonance is a common quantum mechanical phenomenon and it was observed in many systems, as in bulk GaAs in magnetic field, in superlattice 
in electric field, in phonon spectra with electron-phonon coupling. The original paper published in 1961 by Fano [1] was inspired by an asymmetric line shape of an autoionization absorption spectrum of He. His explanation was based on a simple quantum mechanical model, in which there should be possible excitations from a ground state $|g\rangle$ to a continuum of states $\left|\psi_{E}\right\rangle$ as well as to a discrete state $|\phi\rangle$ (see Fig. 2). If both the subsystems are coupled via the matrix element $V_{E}$, their states are modified $\left|\Psi_{E}\right\rangle$ and $|\Phi\rangle$. The profile of the absorption line in the ionization continuum is also changed

$$
\frac{\left|\left\langle\Psi_{E}|T| g\right\rangle\right|^{2}}{\left|\left\langle\psi_{E}|T| g\right\rangle\right|^{2}}=\frac{(q+\epsilon)^{2}}{1+\epsilon^{2}} .
$$

Here, we denoted $\epsilon=\left(E-E_{0}\right) /(1 / 2 \Gamma), \Gamma=2 \pi\left|V_{E}\right|^{2}$ is the broadening of the discrete level $E_{0}$. The parameter $q$ describes the asymmetry of the line shape and is given by the ratio of direct ionization to autoionization

$$
\frac{1}{2} \pi q^{2} \Gamma=\frac{|\langle\Phi|T| g\rangle|^{2}}{\left|\left\langle\psi_{E}|T| g\right\rangle\right|^{2}} .
$$
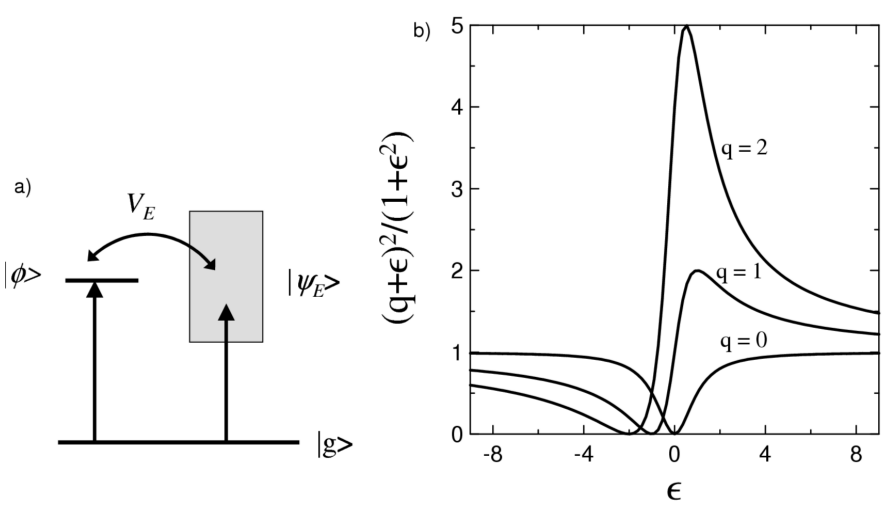

Fig. 2. (a) Scheme for the Fano resonance: electrons are excited from the ground state $|g\rangle$ to the continuum of states $\left|\psi_{E}\right\rangle$, which is coupled with the discrete state $|\phi\rangle$. (b) Profile of the absorption line in the continuum states $(q+\epsilon)^{2} /\left(1+\epsilon^{2}\right)$ for different values of $q$ (reverse the scale of abscissas for negative values of $q$ ).

\subsection{Fano resonance in quantum dot systems}

The Fano resonance was also observed in electronic transport in nanostructures. A first evidence of this effect was observed by a group from MIT in transport through the QD strongly coupled with the electrodes [2]. They measured conductance $\mathcal{G}$ as a function of a gate voltage, which showed asymmetric peaks with a reduction of the conductance below its background value. Their experiment was performed on the device, in which the coupling between the quantum dot and 
the electrodes was tuned by applied voltage. For the weakly coupled QD the single electron sequential tunnelling occurs and the Coulomb blockade effect is observed. For an intermediate strength of the coupling the transmission becomes perfect and the conductance reaches the value $2 e^{2} / h$. The transport characteristics showed features typical of the Kondo resonance - a zero-bias peak and a logarithmic increase in the conductance for $T \rightarrow 0$. When the QD was coupled very strongly to the electrodes the conductance showed a profile typical of the Fano resonance.

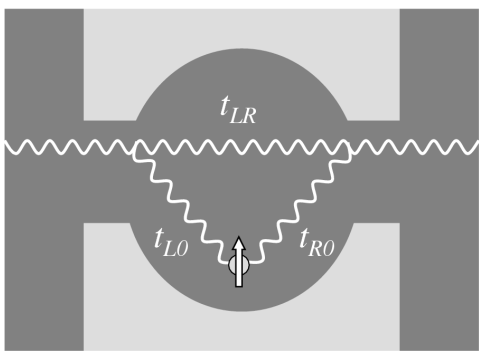

Fig. 3. Presentation of the bridge model for a QD strongly coupled with electrodes. The wave can travel either directly between the electrodes or through impurity states. These channels are called the bridge and the impurity channel. The wave can be multiply scattered in the QD, which leads to the Fano resonance. If an electron is localized on the impurity state, one can expect Fano-Kondo resonance, in which spin-flip processes occur as well.

The MIT experiment was analyzed within the bridge model $[14,15]$. Figure 3 illustrates the waves transmitted through the QD. Due to strong coupling the travelling wave can be directly transmitted between the left and the right electrode (through a bridge channel). In part, however, the wave can be also scattered by localized electrons at the QD. The bridge model fulfils the condition for the Fano resonance, for which the continuum subsystem should be coupled with a discrete state. The waves travelling through the bridge channel belong to the continuum, and the discrete subsystem consists impurity states. The corresponding Hamiltonian is $[14,15]$ :

$$
\begin{aligned}
& H=\sum_{k, \alpha, \sigma} \epsilon_{k \alpha} c_{k \alpha \sigma}^{\dagger} c_{k \alpha \sigma}+\sum_{\sigma} \epsilon_{0} c_{0 \sigma}^{\dagger} c_{0 \sigma}+U n_{0 \uparrow} n_{0 \downarrow} \\
& +\sum_{k, \alpha, \sigma} t_{\alpha 0}\left(c_{k \alpha \sigma}^{\dagger} c_{0 \sigma}+c_{0 \sigma}^{\dagger} c_{k \alpha \sigma}\right)+\sum_{k, k^{\prime}, \sigma}\left(t_{\mathrm{LR}} c_{k \mathrm{~L}, \sigma}^{\dagger} c_{k^{\prime} \mathrm{R}, \sigma}+\text { h.c. }\right),
\end{aligned}
$$

which is an extension of the Anderson model. The last term corresponds to the bridge channel. In the calculations multiple scattering of waves on the contacts have to be included. It means that the electronic wave travelling from the left electrode by the direct channel can be at the right junction backward scattered into the channel with the localized electron. The wave can be traversed to and 
from both the channels in order to get out finally the QD. These multiple scattering processes lead to the Fano-Kondo resonance, which shows features typical of both the resonances.

Assuming quasi-elastic transport, for which the current conservation rule is fulfilled for any energy $E$, one obtains an exact formula for the current [14]:

$$
\begin{aligned}
I= & \frac{2 e}{h} \int \mathrm{d} \omega\left[f_{\mathrm{L}}(E)-f_{\mathrm{R}}(E)\right]\left\{\alpha_{\mathrm{LR}}\left|t_{\mathrm{LR}}\right|^{2}+\operatorname{Im}\left[\alpha_{00}\right] \operatorname{Re}\left[G_{00}^{\mathrm{r}}(E)\right]\right. \\
& \left.+\operatorname{Re}\left[\alpha_{00}\right] \operatorname{Im}\left[G_{00}^{\mathrm{r}}(E)\right]\right\},
\end{aligned}
$$

where $\alpha_{\mathrm{LR}}=4 \pi^{2} \rho^{2} / w^{2}, \alpha_{00}=-4 \pi \rho z_{\mathrm{L}}^{-} z_{\mathrm{L}}^{+*} z_{\mathrm{R}}^{-} z_{\mathrm{R}}^{+*} /\left[w^{2}\left(\left|z_{\mathrm{L}}\right|^{2}+\left|z_{\mathrm{R}}\right|^{2}\right)\right], w=1+$ $\pi^{2} \rho^{2}\left|t_{\mathrm{LR}}\right|^{2}, z_{\mathrm{L}}^{ \pm}=t_{\mathrm{L} 0} \pm \mathrm{i} \pi \rho t_{\mathrm{LR}} t_{\mathrm{R} 0}^{*}, z_{\mathrm{R}}^{ \pm}=t_{\mathrm{R} 0}^{*} \pm \mathrm{i} \pi \rho t_{\mathrm{LR}}^{*} t_{\mathrm{L} 0}$, the electron density of states $\rho$ in the electrodes is assumed to be constant in a small energy range close to the Fermi energy $E_{\mathrm{F}}$, and $G_{00}^{\mathrm{r}}(E)$ denotes the local retarded Green function. The formula (16) includes two new terms: the first one corresponding to the current through the bridge channel and the second one proportional to $\operatorname{Re} G_{00}^{\mathrm{r}}(E)$, which is responsible for the Fano resonance [1]. The third term is proportional to the local density of states $\rho_{0}(E)=-\operatorname{Im}\left[G_{00}^{\mathrm{r}}(E)\right] / \pi$, which leads to the perfect transmission in the Kondo regime. The formula (16) shows also that interference processes play a crucial role in transport and the spin-flip processes are incorporated in modification of the local density of states and formation of the Abrikosov-Suhl resonance.

In calculations we used the equation of motion approach [16] to find the Green function $G_{00}^{\mathrm{r}}(E)$, which were next applied to determine the current and the conductance [14]. The local density of states was slightly modified due to switching on the bridge channel, which confirmed our predictions on a major role of interference in transport. In this system both the resonances, the Fano and the Kondo resonance, are crucial. Figure $4 \mathrm{a}$ presents the best fitting of the conductance to the experimental results [2]. Apart from some quantitative differences the curves show all features observed in the experiment: e.g. an asymmetry between dips and a small bump on the edge of the left dip. The fitting was possible only for a large value of the coupling for the bridge channel $t_{\mathrm{LR}}=320 \mathrm{meV}$, whereas the couplings to the impurity state were much lower $t_{\mathrm{L} 0}=0.6 \mathrm{meV}$ and $t_{\mathrm{R} 0}=4 \mathrm{meV}$. This may be explained by different symmetry of the wave travelling through both the channels. In Fig. 4b we show the profile of the conductance for different asymmetry of the coupling to the left and the right electrode $t_{\mathrm{L} 0} / t_{\mathrm{R} 0}$. The shape of $\mathcal{G}$ depends on the electron number at the QD. The dip on the right hand side of Fig. 4b corresponds to one electron at the QD, whereas the structure on the left hand side - for two electrons. This is a characteristic feature of the Fano-Kondo resonance and it cannot appear for the Fano resonance in absence of the spin-flip processes.

We also performed the analysis of the differential conductance $\mathrm{d} I / \mathrm{d} V_{\mathrm{sd}}$ on the source-drain voltage [14]. Usually in the Kondo limit $\mathrm{d} I / \mathrm{d} V_{\text {sd }}$ shows a zero- 

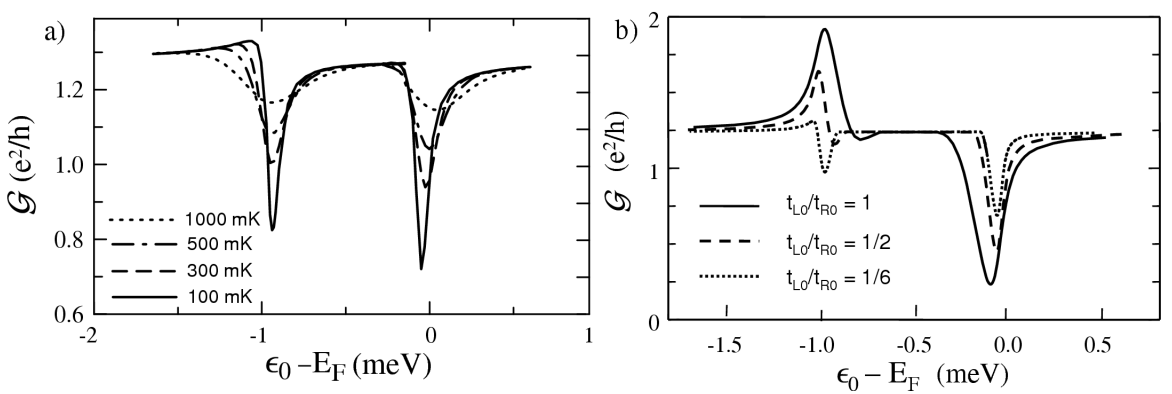

Fig. 4. Conductance $\mathcal{G}$ as a function of the relative position of the dot level $\epsilon_{0}-E_{\mathrm{F}}$ calculated within the bridge model. (a) Our best theoretical fit to the experimental data (Fig. 4a in Ref. [2]) obtained for various temperatures and the parameters $t_{\mathrm{L} 0}=0.6 \mathrm{meV}$, $t_{\mathrm{R} 0}=4 \mathrm{meV}, t_{\mathrm{LR}}=320 \mathrm{meV}, U=1.1 \mathrm{meV}, W=1 \mathrm{eV}$. (b) Change of the profile of the conductance for a different asymmetry of the coupling to the electrodes. The parameters are $t_{\mathrm{R} 0}=4 \mathrm{meV}, t_{\mathrm{L} 0} / t_{\mathrm{R} 0}=1,1 / 2$, and $1 / 6$, the temperature $T=500 \mathrm{mK}$ and the others are the same as in part (a).

-bias peak, which is pinned to the Fermi level. However, in the bridge model the plots of $\mathrm{d} I / \mathrm{d} V_{\text {sd }}$ vs. $V_{\text {sd }}$ showed either a peak or a dip at small voltage. In the experiment [2] the conductance plotted as a grey-scale map showed the diamond structure, but the map was the negative picture of that one observed for the intermediate electrode-QD coupling. It means that the peaks observed in the intermediate coupling were transformed into the dips when the coupling increased. Our studies suggest that the role of the bridge channel becomes more significant for the stronger couplings and it can lead to a such transformation of the conduction profile.

The Fano resonance can occur also in electronic transport through the quantum wire coupled to the side attached QD (see Fig. 5a). The electric current can be in this case simply written as

$$
I=\frac{2 e}{h} \int \mathrm{d} E\left[f_{\mathrm{R}}(E)-f_{\mathrm{L}}(E)\right] \frac{4 \Gamma_{\mathrm{L}} \Gamma_{\mathrm{R}}}{\Gamma_{\mathrm{L}}+\Gamma_{\mathrm{R}}} \operatorname{Im}\left[G_{00}^{\mathrm{r}}(E)\right],
$$

where $\Gamma_{\alpha}=\pi \rho_{\alpha} t_{\alpha 0}^{2}$ is the transfer rate of an electron from the $\alpha$ electrode to the point contact. Destructive interference of the travelling wave with a wave penetrating the QD leads to the modification of the local density of states $\rho_{0}(E)$ at the point contact. This situation is different than that one for the bridge model [let us compare the structure of Eq. (17) and Eq. (16)], where interference processes slightly influenced the Kondo resonance and the local density of states. For the side-attached QD one can expect competition between the Fano and the Kondo resonance, because both the resonances result in a significant modification of the local density of states. The Green function $G_{00}^{\mathrm{r}}(E)$ was calculated in the interpolative perturbation scheme [17], which is reliable for weak and moderate Coulomb 
interactions $U$. Figure 5b presents the plot of $\mathcal{G}$ as a function of the position of the dot level $\epsilon_{1}$. The CB-curves present the weak coupling case, in which the Coulomb blockade effect plays a crucial role. The RR-curves are calculated for a strong coupling, when coherent scattering of the travelling wave on the dot level occurs. In low temperatures $\mathcal{G}$ shows a single broad plateau, whose width is equal to the intra-dot Coulomb interaction $U$. The situation is similar to the Kondo effect for a single level in the QD. The difference is that in the present case $\mathcal{G}$ shows a broad well in contrast to the broad peak seen for the Kondo resonance - therefore, one called the effect as the Kondo antiresonance. Just recently Sato et al. presented preprint [18] with measurements of the conductance in nanowire coupled to the dot. They showed that in weak coupling the conductance shows a series of dips corresponding to a sequential adding of electrons to the QD, which is in analogy to the Coulomb blockade effect. For a stronger coupling the measurements showed that with a decreasing temperature $T \rightarrow 0$ the conductance dips are transformed into the broad well.
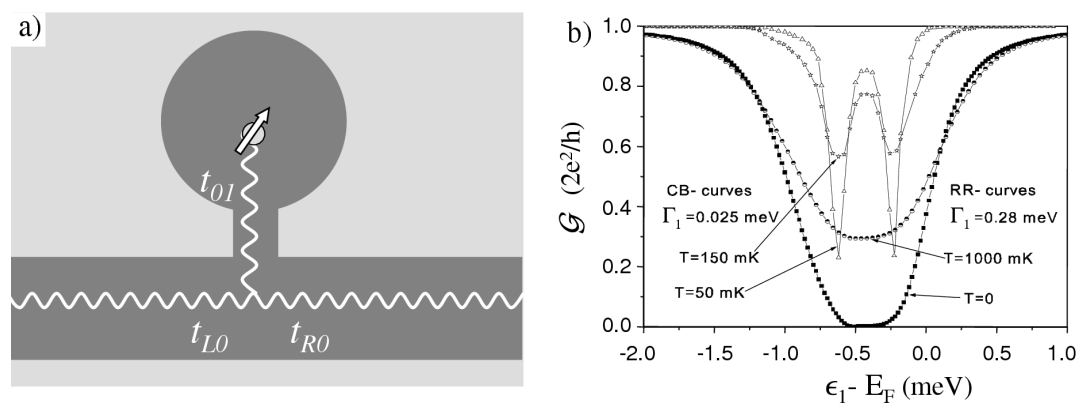

Fig. 5. (a) System with a side-attached QD coupled to the quantum wire and (b) the conductance $\mathcal{G}$ as a function of the relative position of the dot level $\epsilon_{1}-E_{\mathrm{F}}$. The CB-curves are for the weak coupling $\Gamma_{1}=0.025 \mathrm{meV}$ (the Coulomb blockade regime) and the RR-curves for the strong coupling $\Gamma_{1}=0.28 \mathrm{meV}$ (the resonant regime), for which the Fano-Kondo resonance occurs.

A recent experiment performed by Fuhner et al. [19] on a large QD strongly coupled to the electrodes showed the conductance profile as a series of asymmetrical peaks on a very broad maximum. The results indicated on the Fano resonance. On the other hand, Ferry et al. [20] showed that in a large QD one can find a discrete set of pointer states, which are quite stable as the coupling to the environment is increased. The observed pointer states have a narrow line width, and their superposition with nearby quantum states, well coupled to the electrodes, is heavily damped by decoherence processes. In Fig. 6 we present schematically a situation for a large QD with a state available for transmission of an electronic wave, which can be weakly coupled to the discrete set of pointer states. Thus, the system fulfills the condition for the Fano resonance. The model for this case [21] 

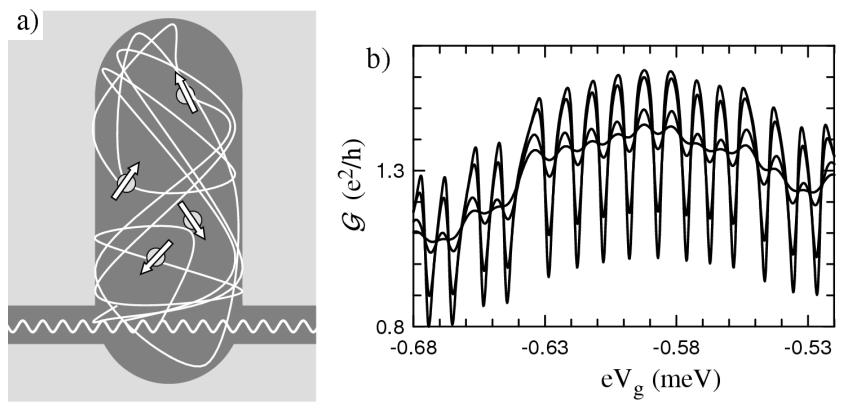

Fig. 6. (a) Presentation of the large QD with a single conducting channel coupled to a set of pointers states. (b) The conductance $\mathcal{G}$ vs. the gate voltage $V_{\mathrm{g}}$ calculated for the model of a semi-open QD. The parameters are taken to get the best fit to the experimental data (Fig. 2b in Ref. [19]) in the temperature range $50 \mathrm{mK} \leq T \leq 1000 \mathrm{mK}$.

is similar to that one for the side-attached QD, where many localized states are taken into account.

The model for this case [21] consists of one very well coupled to the leads localized dot level $\epsilon_{0}$ and a set of sharp in energy scale dot levels $\epsilon_{\gamma}$, which are not directly coupled to the leads. Moreover, $\epsilon_{0}$ is considered as interacting and there is a finite hybridization between $\epsilon_{0}$ and $\epsilon_{\gamma}$ levels. Such the dot configuration possesses the features needed for Fano effect to appear; broad background $\epsilon_{0}$ channel and set of resonant $\gamma$ states hybridized with background channel. However, some features of this resonance are very unusual, very specific to nanodevices. Namely, the quantum destructive interference does not diminish the transmission through the dot down to zero at the Fano dips, as should appear in the classical Fano effect. This is due to localized nature of the background $\epsilon_{0}$ channel and indirect (via $\epsilon_{0}$ ) coupling of the $\gamma$ states to the electrodes. Moreover, strong electron correlations (the Kondo effect) present within $\epsilon_{0}$ suppress the fluctuations of the particle number due to the Fano resonance.

\subsection{Aharonov-Bohm effect and Fano resonance}

The Aharonov-Bohm effect [3] is another example showing the wave nature of electrons in presence of magnetic field. The original idea by Aharonov and Bohm was to perform an interference experiment, in which a beam of electrons is split into two and they are sent around a long solenoid. Since the solenoid is very long, the intensity of the magnetic field $\boldsymbol{B}$ is very small, but the electronic waves are affected by the vector potential $\boldsymbol{A}$. There is a shift of the phase of the electronic wave $\phi_{\Gamma}=(e / \hbar) \int_{\Gamma} \boldsymbol{A} \cdot \mathrm{d} \boldsymbol{s}$, which depends on a trajectory $\Gamma$. The electronic wave passing clockwise around the solenoid has a different phase shift than that one passing counter-clockwise. The interference pattern depends on the magnetic flux $\Phi$. A first observation of the Aharonov-Bohm effect in condensed matter was performed by Webb et al. [4] in 1985 on the metallic ring of the diameter of 
a few $\mu \mathrm{m}$. They measured the conductance, which was dependent on the magnetic flux $\Phi$ enclosed in the ring and oscillated with the period equal to the flux quantum $\Phi_{0}=h c / e$. Nowadays one can produce metallic rings with a quantum dot in one of the arm [5]. Such a system allows to study quantum interference together with electronic correlations manifested by the Kondo resonance.

The bridge model is also suitable for studies of the electronic transport through the metallic ring with the Kondo impurity. In the Hamiltonian (15) the hopping integrals have to be taken as complex numbers $t_{\nu}=\left|t_{\nu}\right| \mathrm{e}^{\mathrm{i} \phi_{\nu}}$, where $\phi_{\nu}$ corresponds to the shift of the phase of electronic wave passing through the $\nu$ arm of the ring $(\nu=\mathrm{LR}, \mathrm{L}, \mathrm{R})$ in presence of the magnetic field. The shift of the phase is related to the magnetic flux enclosed in the ring $2 \pi \Phi / \Phi_{0}=\phi_{\mathrm{LR}}-\phi_{\mathrm{L}}-\phi_{\mathrm{R}}$. At $T=0$ one can derive the zero-bias conductance from Eq. (16), which simplifies in the Kondo regime (for the number of electrons $n \rightarrow 1$ ) to the form

$$
\mathcal{G}=\frac{2 e^{2}}{h} \frac{4\left|t_{\mathrm{L}}\right|^{2}\left|t_{\mathrm{R}}\right|^{2}}{\left(\left|t_{\mathrm{L}}\right|^{2}+\left|t_{\mathrm{R}}\right|^{2}\right)^{2} w^{2}}\left[1+\pi^{4} \rho^{4}\left|t_{\mathrm{LR}}\right|^{4}-2 \pi^{2} \rho^{2}\left|t_{\mathrm{LR}}\right|^{2} \cos \left(2 \pi \Phi / \Phi_{0}\right)\right]
$$

As expected, $\mathcal{G}$ oscillates with the period $\Phi_{0}=h c / e$. Moreover, $\mathcal{G} \rightarrow 0$ for $t_{\mathrm{L}} \rightarrow 0$, which means that the transport through the system becomes blocked. For the case $t_{\mathrm{L}}=0$ there is no electronic transport through the QD, although it is connected with the right electrode $t_{\mathrm{R}} \neq 0$. Due to coherent electronic correlations between the wave travelling through the bridge channel and multiple scatterings on the $\mathrm{QD}$, the transmission through the bridge channel is reduced and in the Kondo regime is completely blocked.

For finite temperature $T$ and a source-drain voltage $V_{\text {sd }}$ we determined numerically the non-equilibrium Green function and the current. The results for the differential conductance $\mathrm{d} I / \mathrm{d} V_{\mathrm{sd}}$ are presented in Fig. 7 for various values of the
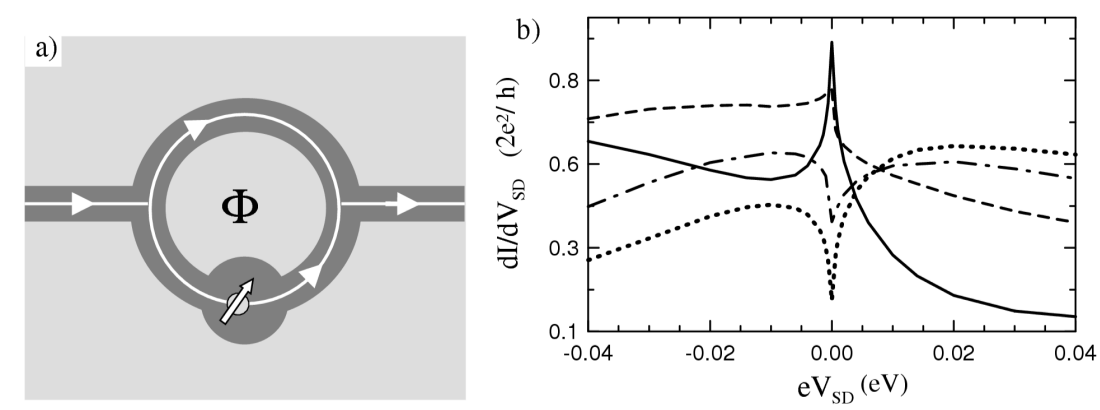

Fig. 7. (a) Schematic presentation of the Aharonov-Bohm effect for the metallic ring with a quantum dot in the arm and (b) the differential conductance $\mathrm{d} I / \mathrm{d} V_{\text {sd }}$ as a function of the source-drain voltage $V_{\text {sd }}$ for various magnetic flux $\Phi$ enclosed within the ring $\Phi=0.5 h c / e$ (dotted), 0.25hc/e (dash-dotted), 0.125hc/e (dashed) and 0 (solid). In the calculations $t_{\mathrm{L} 0}=0.6 \mathrm{meV}, t_{\mathrm{R} 0}=4 \mathrm{meV}, t_{\mathrm{LR}}=320 \mathrm{meV}, W=1 \mathrm{eV}$, the temperature $T=500 \mathrm{mK}$ and $\epsilon_{0}-E_{\mathrm{F}}=-6 \mathrm{meV}$. 
flux $\Phi$ enclosed in the ring. It is seen that the curves show either a peak or a dip at $V_{\mathrm{sd}}=0$. This is an evidence of either a constructive or a destructive interference process between electronic waves travelling through the ring in the presence of the Kondo resonance. We propose to perform an experiment, in which one can observe a continuous evolution of the shape of $\mathrm{d} I / \mathrm{d} V_{\text {sd }}$ with a change of the flux $\Phi$ - the zero-bias peak should be transformed into the dip. This effect can be seen only for the QD in the Kondo regime $\left(\epsilon_{0} \ll \epsilon_{\mathrm{F}}\right)$. If the energy level $\epsilon_{0}$ is shifted to the empty state regime $\left(\epsilon_{0} \gg \epsilon_{\mathrm{F}}\right)$, and the electronic transport is uncorrelated, the differential conductance does not show any structure around $V_{\text {sd }}=0$.

\section{Final remarks and summary}

In this paper we have presented two mechanisms relevant for electronic transport in nanostructures: quantum interference and Coulomb interactions. A few examples of systems were described, in which the Fano resonance as special type of interference between the travelling wave and a set of localized states was observed. The profile of the conductance has dips as a signature of destructive interference. The conditions for the Fano resonance can be found in many other nanostructures. Figure 8 presents the scheme of an experiment performed by Wrobel et al. [22] for

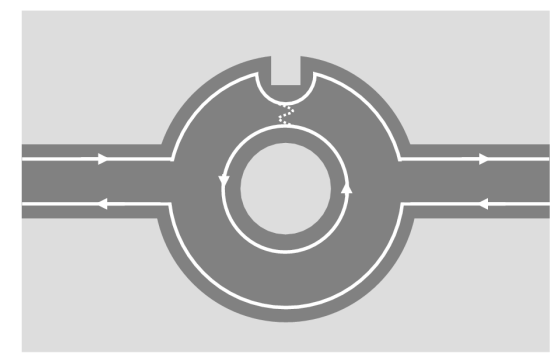

Fig. 8. Scheme of electronic transport through the metallic ring in a strong magnetic field with two edge currents: in the inner diameter and the outer diameter of the ring (after [22]). The conditions for the Fano resonance are fulfilled as the constriction results in coupling of the conducting channel current with the localized state.

electronic transport through the metallic ring in a magnetic field, which is very strong and results in quantization of electronic motion. In this case two edge currents flow in the ring: in the outer and in the inner part. The current in the outer diameter is connected with the conducting channel and it can be detected in a conductance measurement. The current in the inner part of the ring is disconnected from the electrodes, but charge accumulated in it acts on the conducting channel and leads to the Coulomb blockade effect [22]. In this system the conditions for the Fano resonance are fulfilled and the effect can be observed when both the currents are coupled. The authors intentionally introduced a constriction in the outer part of the ring, which disturbed a current flow. The transport measurements showed 
additional dips in the conductance plots, which was interpreted as the Coulomb blockade effect [22]. In our opinion, the constriction introduces a coupling between the conducting channel and the localized state (the currents in the outer and the inner part) and the appearance of the dips is an evidence for the Fano resonance.

We also considered a role of the Coulomb interactions resulting in many-body effects like the Kondo resonance, which is caused by conducting electrons resonantly scattered by localized spins. In nanostructures the characteristic feature of the Kondo resonance is an increase in the conductance for $T \rightarrow 0$, whereas in the bulk systems one observes an increase in the resistance. Electronic transport in nanostructures has to be considered within the Landauer approach, in which the one-dimensional geometry is relevant for scattering processes and leads to perfect transmission in the Kondo regime at $T \rightarrow 0$. We showed the interplay of the Kondo and the Fano resonance. In the bridge model the additional channel, which directly couples the electrodes, can change the profile of the conductance curve. The conductance peaks, features characteristic of the Kondo resonance, are transformed to the dips. The same model is applied to the metallic ring with the QD in an arm. The zero bias peak is continuously transformed to the dip with an increase in the magnetic flux enclosed in the ring. Then for the dot side-attached to the quantum wire the situation is different, and the Kondo resonance competes with the Fano resonance. In low temperatures the Kondo resonance dominates and suppresses the conductance oscillations due to the Fano effect.

\section{Acknowledgments}

We would like to express our acknowledgement to Tomasz Kostyrko for discussion and his help during writing the paper. The work was supported by the Ministry of Science and Information Society Technologies and RTNNANO contract No. MRTN-CT-2003-504574.

\section{References}

[1] U. Fano, Phys. Rev. 124, 1866 (1961).

[2] J. Gores, D. Goldhaber-Gordon, S. Heemeyer, M.A. Kastner, H. Shtrikman, D. Mahalu, U. Meirav, Phys. Rev. B 62, 2188 (2000).

[3] Y. Aharonov, D. Bohm, Phys. Rev. 115, 485 (1959).

[4] R. Webb, S. Washburn, C. Umbach, R. Laibowitz, Phys. Rev. Lett. 54, 2696 (1985).

[5] A. Yacoby, M. Heiblum, D. Mahalu, H. Shtrikman, Phys. Rev. Lett. 74, 4047 (1995); U. Gerland, J. von Delft, T.A. Costi, Y. Oreg, Phys. Rev. Lett. 84, 3710 (2000); W.G. van der Wiel, S. De Franceschi, T. Fujisawa, J.M. Elzerman, S. Tarucha, L.P. Kouwenhoven, Science 289, 2105 (2000); K. Kobayashi, H. Aikawa, S. Katsumoto, Y. Iye, Phys. Rev. Lett. 88, 256806 (2002); U.F. Keyser, C. Fuhner, S. Borck, R.J. Haug, M. Bichler, G. Abstreiter, W. Wegscheider, Phys. Rev. Lett. 90, 196601 (2003); U.F. Keyser, C. Fuhner, R.J. Haug, W. Wegscheider, M. Bichler, G. Abstreiter, Phys. Status Solidi B 238, 331 (2003); H. Aikawa, K. Kobayashi, A. Sano, S. Katsumoto, Y. Iye, Physica E 22, 474 (2004). 
[6] J. Kondo, Prog. Theor. Phys. 32, 37 (1964).

[7] L.I. Glazman, M.E. Raikh, JETP Lett. 47, 452 (1988).

[8] T.K. Ng, P.A. Lee, Phys. Rev. Lett. 61, 1768 (1988).

[9] A. Kawabata, J. Phys. Soc. Jpn. 60, 3222 (1991).

[10] L. Kouwenhoven, L.I. Glazman, Phys. World 14, 33 (2001).

[11] D. Goldhaber-Gordon, J. Göres, M.A. Kastner, H. Shtrikman, D. Mahalu, U. Meirav, Phys. Rev. Lett. 81, 5225 (1998); D.C. Ralph, R.A. Buhrman, Phys. Rev. Lett. 72, 3401 (1994); S.M. Cronenwett, T.H. Oosterkamp, L.P. Kouwenhoven, Science 281, 540 (1998); L.P. Rokhinson, L.J. Guo, S.Y. Chou, D.C. Tsui, Phys. Rev. B 60, 16319 (1999); L. Kouwenhoven, L.I. Glazman, Phys. World 14, 33 (2001).

[12] J. Nygard, D.H. Cobden, P.E. Lindelof, Nature 408, 342 (2000).

[13] W.J. Liang, M.P. Shores, M. Bockrath, J.R. Long, H. Park, Nature 417, 725 (2002); J. Park, A.N. Pasupathy, J.I. Goldsmith, C. Chang, Y. Yaish, J.R. Petta, M. Rinkoski, J.P. Sethna, H.D. Abruna, P.L. McEuen, D.C. Ralph, Nature 417, 722 (2002).

[14] B.R. Bułka, P. Stefański, Phys. Rev. Lett. 86, 5128 (2001).

[15] S. Heemeyer, Ph.D. thesis, Massachusetts Institute of Technology, 2000.

[16] C. Lacroix, J. Phys. F 11, 2389 (1981).

[17] P. Stefanski, Solid State Commun. 128, 29 (2003).

[18] M. Sato, H. Aikawa, K. Kobayashi, S. Katsumoto, Y. Iye, arXiv:condmat/0410062, v2 21 Feb 2005.

[19] C. Fuhner, U.F. Keyser, R.J. Haug, D. Reuter, A.D. Wieck, Phys. Status Solidi $C$ 0, 1305 (2003).

[20] D.K. Ferry, R. Akis, J.P. Bird, Phys. Rev. Lett. 93, 026803 (2004).

[21] P. Stefański, A. Tagliacozzo, B.R. Bułka, Phys. Rev. Lett. 93, 186805 (2004).

[22] J. Wrobel, T. Dietl, K. Reginski, M. Bugajski, Phys. Rev. B 58, 16252 (1998). 